\title{
Comparison of screen-detected and interval colorectal cancers in the Bowel Cancer Screening Programme
}

\author{
MD Gill',1,2,3, MG Bramble ${ }^{2,3}$, CJ Rees ${ }^{2,3}$, TJW Lee ${ }^{2,4}$, DM Bradburn' and SJ Mills' \\ 'Northem Colorectal Cancer Audit Group, Northumbria Health Care Trust, Northumberland, UK; ${ }^{2}$ Northem Region Endoscopy Group, Stockton on Tees, UK; \\ ${ }^{3}$ Wolfson Research Institute, Durham University Queen's Campus, Stockton on Tees, UK; ${ }^{4}$ Institute of Health and Society, Newcastle University, \\ Newcastle upon Tyne, UK
}

BACKGROUND: The NHS Bowel Cancer Screening Programme (BCSP) offers biennial faecal occult blood testing (FOBt) followed by colonoscopy after positive results. Colorectal cancers (CRCs) registered with the Northern Colorectal Cancer Audit Group database were cross-referenced with the BCSP database to analyse their screening history.

METHODS: The CRCs in the screening population between April 2007 and March 2010 were identified and classified into four groups: control (diagnosed before first screening invite), screen-detected, interval (diagnosed between screening rounds after a negative FOBt), and non-uptake (declined screening). Patient demographics, tumour characteristics and survival were compared between groups.

RESULTS: In all, 5 I I out of I 336 (38.2\%) CRCs were controls; 825 (61.8\%) were in individuals invited for screening of which 322 (39.0\%) were screen detected, 3 II (37.7\%) were in the non-uptake group, and I 92 (23.3\%) were interval cancers. Compared with the control and interval cancer group, the screen-detected group had a higher proportion of men $(P=0.002, P=0.003$ respectively), left colon tumours $(P=0.007, P=0.003$ ), and superior survival (both $P<0.001$ ). There was no difference in demographics, tumour location/stage, or survival between control and interval groups.

CONCLUSION: The FOBt is better at detecting cancers in the left colon and in men. The significant numbers of interval cancers weren't found to have an improved outcome compared with the non-screened population.

British Journal of Cancer (2012) 107, 4I7-421. doi:10.1038/bjc.2012.305 www.bjcancer.com

Published online 10 July 2012

(C) 2012 Cancer Research UK

Keywords: colorectal neoplasm; screening; colorectal cancer; colonoscopy

Colorectal cancer (CRC) is a major public health problem. It is estimated that men have a 1 in 16 and women a 1 in 20 lifetime risk of being diagnosed with CRC. In 2007-2008, there were $\sim 17100$ new cases diagnosed in men and 14400 in women, making it the third most commonly diagnosed cancer (excluding non-melanoma skin cancers) in the United Kingdom. In the same time period, $\sim 13300$ people died from CRC, the second highest cancer mortality rate in the United Kingdom (Cancer Research UK, 2010).

The NHS Bowel Cancer Screening Programme (BCSP) was introduced in April 2006 to decrease the mortality from CRC. The primary aim of the programme is to detect CRC at an earlier stage, thereby improving both the morbidity and prognosis of patients. A secondary aim is to identify and remove adenomas.

In the north east of England, full uptake of the screening programme was achieved by April 2008. The population eligible for screening includes anyone aged 60-69 years, extended to 74 years in January 2010. Screening comprises biennial nonrehydrated guaiac-based faecal occult blood tests (FOBts) (Hemascreen, Immunostics Inc., Ocean, NJ, USA). The test requires completion of six windows from three consecutive stool specimens. If five or six are positive the test is abnormal and colonoscopy is offered to the individual. If one to four windows are positive the test is defined as unclear and up to two repeat tests are

*Correspondence: MD Gill; E-mail: m.gill@nhs.net

Received 24 February 2012; revised 8 June 2012; accepted 18 June 20 I2; published online 10 July 2012 undertaken. If both of these are clear the screening episode is considered to be negative. If any further windows are positive this is classified as a weak positive result and the patient will be offered a colonoscopy. Colonoscopy within the BCSP is performed only by accredited endoscopists who undergo continuous audit to ensure a consistently high standard of colonoscopy (Lee et al, 2012a).

Within any cancer type covered by a screening programme there are three distinct categories of cancer diagnoses. These are nonuptake cancers (the population who decline the screening test that are later diagnosed with a cancer), interval cancers (a cancer diagnosed between screening rounds, after a negative screening episode) and screen-detected cancers. An interval cancer may be diagnosed after a normal FOBt or screening colonoscopy, and before the screened individual was due to undergo their next planned screening test (FOBt or surveillance colonoscopy). Within our study, we define a fourth control group: patients diagnosed with a CRC before their completion of a screening episode. They belong to the same age range and were diagnosed in the same time frame as those offered screening.

Interval cancers may either be due to a false-negative screening test result, or aggressive, fast-growing tumours that have developed between screening rounds. Possible causes of increased aggressiveness of these tumours include increased levels of microsatellite instability from loss of function of mismatch repair genes (Sawhney et al, 2006), and positive cytosinephosphate-guanine island methylator phenotype (CIMP) status (Arain et al, 2010). 
The aims of the study were to identify all CRCs, in the population eligible for screening, comparing the patient demographics, tumour stage/site, and short-term outcomes between the four groups defined above. Secondary aims were to look in detail at the effectiveness of the FOB test.

\section{MATERIALS AND METHODS}

\section{Trial design and participants}

The Northern Colorectal Cancer Audit Group (NORCCAG) has collected data on all patients with CRC within the North East of England continuously since 1997. It covers a population of $\sim 3.1$ million. NORCCAG collects patient demographics, radiological, endoscopic, surgical, oncological, and pathological data for each new diagnosis that is discussed in dedicated colorectal multidisciplinary team meetings. Dates of death are collected via the Office of National Statistics. Over this study period, data were collected directly from each unit, as well as from the National Bowel Cancer Audit Project (NBOCAP). The 2010 NBOCAP annual report described case ascertainment of $\sim 81 \%$ for the North of England cancer network (National Bowel Cancer Audit Project, 2011).

The study design and methodology was endorsed by members of the Northern Region Endoscopy Group, a collaborative research network representing over 300 endoscopists (Rees and Rutter, 2010).

The NORCCAG database was interrogated to identify all CRCs diagnosed between April 2007 and March 2010, in patients aged between 60-69 years. Details of relevant investigation, surgical, and pathological data were extracted for each patient. Patients living outside the North East screening hub region were excluded.

This patient group was then cross-referenced with the regional bowel cancer screening database to gain the relevant screening history for each patient. These data included details of FOB invitations and results, screening nurse practitioner appointments, and details of screening colonoscopies.

The two databases were combined to allow classification of patients into their respective groups of control, interval, nonuptake, and screen-detected cancers. Patients who declined the FOB test $(n=301)$, or declined a screening nurse practitioner appointment $(n=3)$, and those who declined a screening colonoscopy $(n=7)$ were combined into one non-uptake group. All patients that submitted a positive FOB result, but were diagnosed through symptomatic bowel cancer services before completion of a full screening round, were classified into the control group $(n=19)$, along with the population who were diagnosed before receiving their first screening invite $(n=492)$.

As the study is classified as service evaluation, formal ethical approval was not required. Confirmation of this was given by the local NHS research and ethics committee. The National Information Governance Board was approached who accepted that pseudoanonymisation of patients between databases was sufficient for formal approval not to be required.

\section{Data analysis}

American Society of Anaesthesiologists (ASAs) physical status grade was used as a surrogate for severity of patient comorbidities, being dichotomised into two groups of $1-2$ and 3-5. Tumour location was dichotomised on being distal to the splenic flexure or proximal (up to and including the splenic flexure). The deprivation level was calculated using the participants' postcode to derive their respective index of multiple deprivation rank (2007) for the Lower Super Output Area that they reside in. This was then dichotomised into two groups; whether or not the patient lived in the $10 \%$ most deprived areas within England. The modified Dukes' staging classification (stages A to D) was used to stage each tumour. Local excisions included all endoscopic polypectomies and trans-anal resection of tumours. Pearson $\chi^{2}$ tests were used for variables of deprivation, gender, ASA grade, and tumour location. KruskalWallis test was used for Dukes stage as an ordinal variable, with log-rank Mantel-Cox test used for survival analysis. Data were analysed using SPSS version 19.0 (SPSS Inc., Chicago, IL, USA). Significance in analysis was taken at the 0.05 level.

\section{RESULTS}

Within the study period of 1 April 2007 to 31 March 2010, 954305 screening invitations were distributed, with an uptake rate of $56.0 \%$. Colorectal cancers were diagnosed in 1336 patients within the age range eligible for screening. Of these, $511(38.2 \%)$ were in patients before receiving their first screening invite (our control group), and $825(61.8 \%)$ were diagnosed in those offered screening. Of those offered screening, $311(37.7 \%)$ were in patients who didn't complete the screening process (non-uptake), $192(23.3 \%)$ were interval cancers, and $322(39.0 \%)$ screen-detected cancers.

Those with unknown tumour stage due to the patient being unfit or declining surgical resection $(n=25)$, missing tumour stage $(n=1)$, or those with no evidence of tumour found at surgical resection after chemo/radiotherapy $(n=14)$ were not included in the analysis using Dukes stage as a variable.

\section{Screen-detected cancers $v s$ control group}

When compared with the control group, screen-detected cancers were found predominantly in men and in the left colon, with no difference in ASA grade or deprivation level (Table 1). Significantly more Dukes' A and fewer Dukes' D cancers were detected, with a superior short-term crude survival (all cause, log-rank Mantel-Cox $\chi^{2}=53.617$, d.f. $\left.=1, P<0.001\right)$. Significantly more local excisions were performed in the screen-detected group (for all stages of tumour and for Dukes' A cancers) compared with control, interval, and non-uptake groups $(P<0.01)$, as well as significantly fewer palliative procedures and cases with no intervention $(P<0.05)$ (Table 2).

\section{Screen-detected cancers $v s$ interval cancers}

When compared with the interval cancers, screen-detected cancers were found predominantly in men, with no difference in ASA grade or deprivation level (Table 1). Screen-detected cancers were predominantly in the left colon with significantly more Dukes' A, fewer Dukes' D cancers, and a superior short-term crude survival (all cause, log-rank Mantel-Cox $\chi^{2}=50.361$, d.f. $=1, P<0.001$ ).

\section{Positivity of the FOBt}

There were a total of 551 individuals that were diagnosed with a CRC who had returned at least one FOBt kit. In all, 339 (61.5\%) were positive test results, $36.8 \%$ were negative test results, $8(1.5 \%)$ were incomplete kits (all returned one unclear test), and $1(0.2 \%)$ was a spoilt kit unsuitable to be read.

Comparing the negative $v s$ positive test results, on univariate logistic regression analysis, male gender $(P=0.022)$, tumours distal to the splenic flexure $(P=0.001)$ and earlier cancers (Dukes stage $A / B, P<0.001)$ were predictors of a positive $F O B$ result. These remained significant on multivariate analysis $(P=0.013$, $P=0.005$, and $P<0.001$, respectively).

\section{Interval cancers $v s$ control group}

When compared with the control group, there was no significant difference found in gender, ASA grade, deprivation level, tumour site, Dukes stage, operative management, or short-term survival (log-rank Mantel-Cox $\chi^{2}=0.478$, d.f. $=1, P=0.489$ ). 
Table I Age analysed using t-test

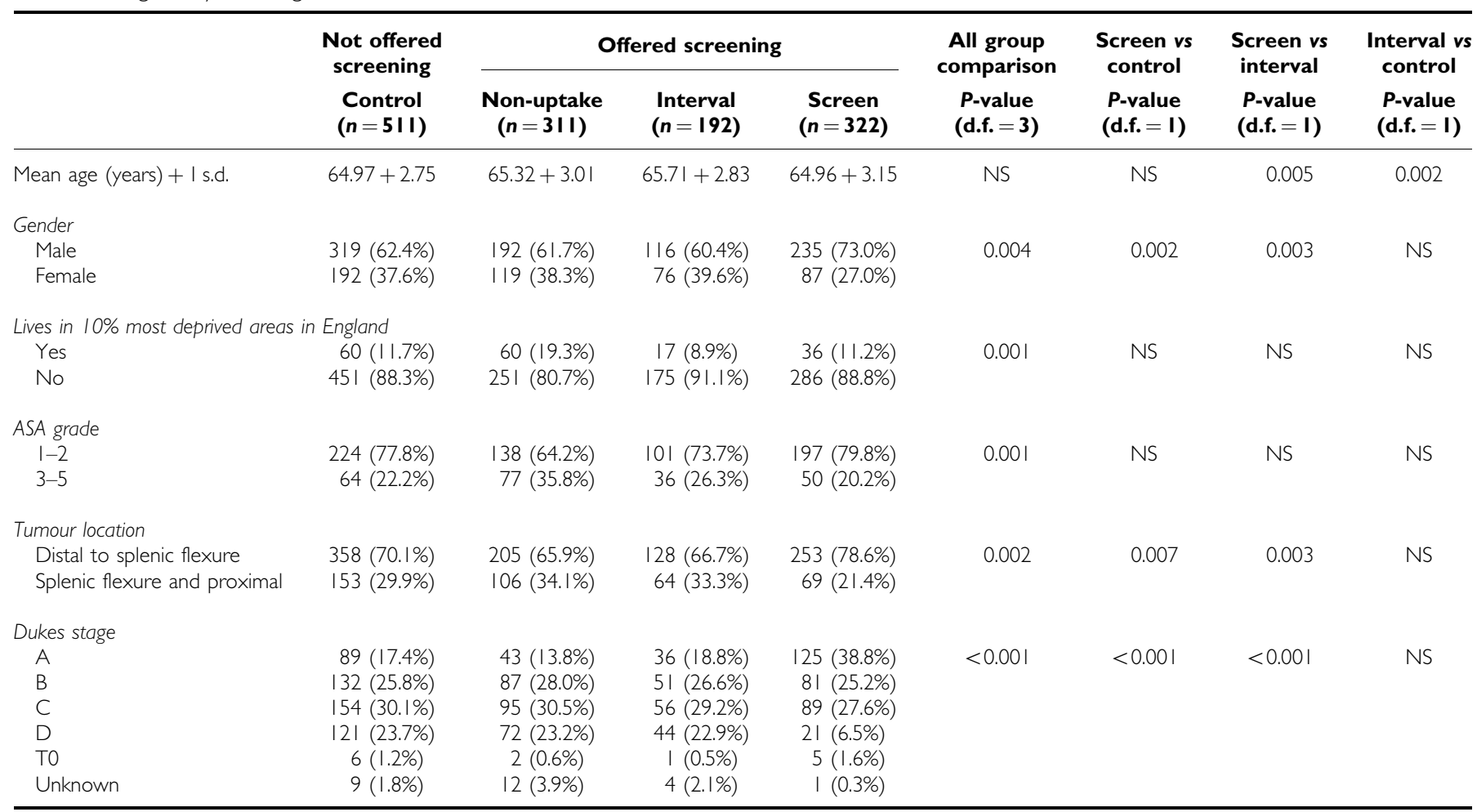

Abbreviations: ASA = American Society of Anaesthesiologist; NS = not significant; s.d. = standard deviation. Gender, ASA grade, deprivation level, and tumour location analysed using $\chi^{2}$ test; Dukes stage analysed using Kruskal-Wallis test. ASA grade was recorded in $66.4 \%$ of cases.

Table 2 Operative type by group, all cases

\begin{tabular}{|c|c|c|c|c|}
\hline \multirow[b]{2}{*}{$\begin{array}{l}\text { Operative } \\
\text { type }\end{array}$} & \multirow{2}{*}{$\begin{array}{c}\text { Not offered } \\
\text { screening } \\
\text { Control } \\
(n=5 I I)\end{array}$} & \multicolumn{3}{|c|}{ Offered screening } \\
\hline & & $\begin{array}{c}\text { Non-uptake } \\
(n=3 I I)\end{array}$ & $\begin{array}{l}\text { Interval } \\
(n=192)\end{array}$ & $\begin{array}{c}\text { Screen } \\
(n=322)\end{array}$ \\
\hline Resective & 405 (79.3\%) & $240(77.2 \%)$ & $152(79.2 \%)$ & $274(85.1 \%)$ \\
\hline Local excision & $15(2.9 \%)$ & $8(2.6 \%)$ & $4(2.1 \%)$ & 38 (11.8\%) \\
\hline Palliative procedure & $33(6.5 \%)$ & $26(8.4 \%)$ & $10(5.2 \%)$ & $3(0.9 \%)$ \\
\hline No procedure & $58(11.4 \%)$ & 37 (11.9\%) & $26(13.5 \%)$ & 7 (2.2\%) \\
\hline
\end{tabular}

\section{FOB test results from previous screening rounds}

There were 46 cases where the patient had submitted a FOB test from a screening round before the round in which their cancer was diagnosed. A total of 32 patients submitted a normal FOBt at their first screening round, with 26 being diagnosed with a screendetected cancer and 6 an interval cancer after their next round of screening. Of the 32 earlier normal tests, 14 (43.8\%) were of Dukes stage $C$ or D; 12 patients declined screening at their first offer, with 11 being diagnosed with a screen-detected cancer and 1 an interval cancer at the subsequent screening round.

\section{Comparison of subgroups of interval cancers}

In all, $15(7.8 \%)$ interval cancers had one unclear FOB test first before having two normal tests. Compared with the interval cancer group with one normal test, there was no difference found in gender $\left(\chi^{2}=1.286\right.$, d.f. $\left.=1, P=0.257\right)$, ASA grade $\left(\chi^{2}=0.007\right.$, d.f. $=1, \quad P=0.933), \quad$ deprivation level $\left(\chi^{2}=2.505, \quad\right.$ d.f. $=1$, $P=0.114)$, tumour location $\left(\chi^{2}=1.302\right.$, d.f. $\left.=1, P=0.254\right)$, Dukes stage $\left(\gamma^{2}=0.055\right.$, d.f. $\left.=1, P=0.815\right)$, or survival (log-rank MantelCox, $\left.\chi^{2}=1.638, P=0.201\right)$. Those with one unclear test were diagnosed with their cancer significantly sooner (7.5 vs 12.8 months from completion of the FOB test, $t=2.987, P=0.003)$ to those with one normal test.

\section{Survival}

Dates of death before 1 November 2011 were recorded. Mean follow-up for all cases was 29.3 months (range 0-54.9 months, s.d. 15.1 months).

There was a significant difference in survival between groups (log-rank Mantel-Cox, $\chi^{2}=69.018$, d.f. $=3, P<0.001$ ) when the screen-detected group is compared against all other groups, both individually and combined as shown in Figure 1 . When the control, non-uptake, and interval cancer groups are compared against each other, no significant difference was found in survival.

\section{DISCUSSION}

This is a comprehensive study of CRC in one English region following the implementation of the National BCSP. By using the combination of the NORCCAG database and the BCSP database, an accurate comparison of all cancers and their short-term outcomes has been demonstrated.

Clinical population studies from Nottingham, Funen, Minnesota, Burgundy, and Goteborg (Kronborg et al, 1987; Kewenter et al, 1994; Hardcastle et al, 1996; Kronborg et al, 1996; Mandel et al, 1999; Faivre et al, 2004) have shown that screening increases the detection of earlier CRCs, accompanied by an overall improvement in survival for screen-detected cancers. Pilot studies performed by Hardcastle et al. in Nottingham between 1981 and 1991 showed that there was a $15 \%$ reduction in cumulative CRC mortality in the screening group. There was also a larger proportion being diagnosed with earlier bowel cancers (Dukes A). Of the 893 cancers diagnosed in the group offered screening 


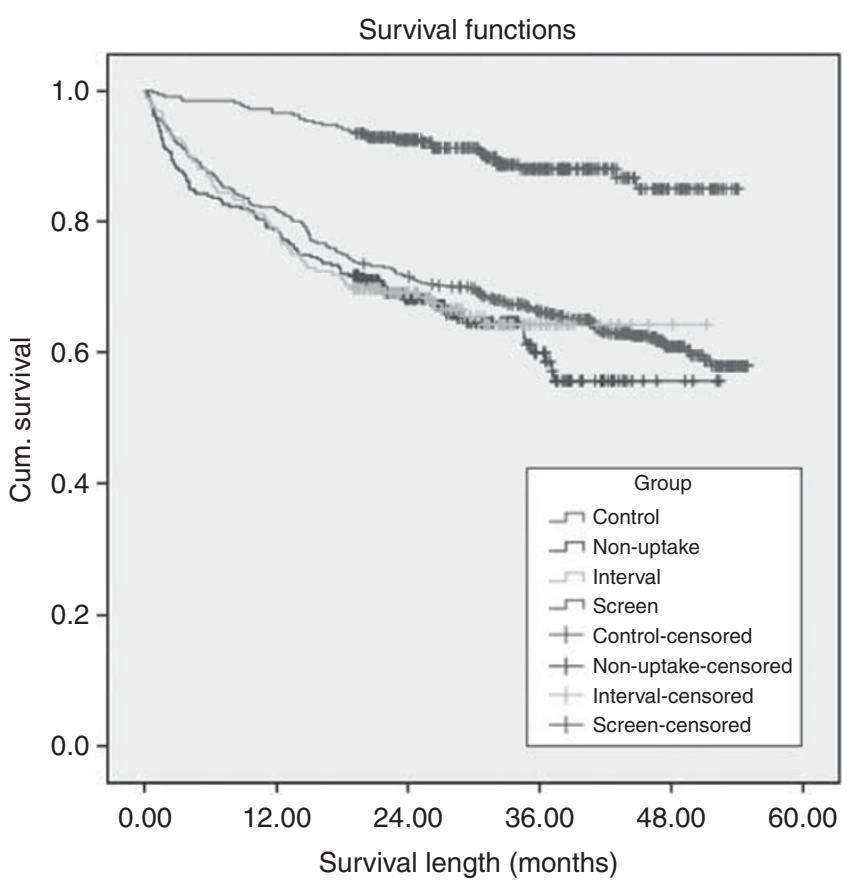

Figure I Survival curves, all cause mortality.

(CRC incidence of 1.49 per 1000 person-years), 236 (26.4\%) were detected by faecal occult blood (FOB) screening, 249 (27.9\%) presented after a negative FOB test or investigation, and 400 (44.8\%) presented in nonresponders (Hardcastle et al, 1996). Compared with these results, our study shows that the proportion of cancers detected through screening has improved with implementation of the national programme, with a decrease in the proportion in nonresponders. This could be due to improved awareness of the screening programme through national media campaigns. Our study also shows that nearly $40 \%$ of all screendetected cancers are Dukes stage A with an improved survival rate compared with the non-screen-detected cancer population. The earlier stage profile in screen-detected cancers may represent the true prevalence of CRCs within a population. The natural history of CRCs and the asymptomatic nature of early cancers mean that more advanced tumours are more likely to present through symptomatic services, as well as being present for a shorter duration before progressing to a more advanced stage (i.e., metastasising to nodes/distant organs).

Factors influencing the positivity of the non-rehydrated guaiac FOBt in the detection of CRC can be analysed by comparing the patient and tumour characteristics of positive and negative test results. Left-sided lesions and male sex were associated with significantly greater proportions of positive FOB results.

A recent paper by Steele et al (2012) reviewed the rates and outcomes of interval cancers within the pilot study of the Scottish Bowel Cancer Screening Programme. They also found that interval cancers were detected more frequently in women and in the right colon. Reasons for the difference due to gender are unclear. One possible explanation may be differing levels of oestrogen in women, with earlier stage tumours having been associated with the use of hormonal therapy (Chlebowski et al, 2004). Combined hormonal therapy has also been used in treatment of angiodysplasia with its effects of improved endothelial integrity and shortened bleeding time. However, trial results using this therapy have been mixed (Dray et al, 2011). With the median age of natural menopause being 49 years in a UK cohort (interquartile range 45.0-51.0) (Pokoradi et al, 2011), the vast majority of women in the screening population will be post-menopausal. The role of medications which affect hormone levels (e.g., HRT) is unknown and may benefit from further analysis. Similarly, although aspirin use has been shown to be associated with a lower neoplasia detection rate in patients with a positive FOBt, its effect on the positivity of the FOBt in those with a CRC has not been established (Lee et al, 2012b).

Although Steele et al (2012) found improved survival in patients with interval cancers over the non-screened control group, there was no significant difference in survival between these two groups in our study. When interval cancers were compared against the population who were offered but did not take up screening, and also against the control group, there was no difference found between Dukes stage or survival. This finding might be anticipated given that all interval cancers presented symptomatically, therefore similar tumour characteristics and outcomes as the control (nonscreened group) would be expected. It does not support the suggestion that aggressive, fast-growing tumours may disproportionately present as interval cancers after a false-negative FOB test result.

In all, $7.8 \%$ of interval cancers were found in patients who had returned one unclear kit (one to four windows positive) followed by two normal tests, giving an overall negative result. Although this subgroup of cancers showed no significant difference in patient demographics, tumour stage, or survival, this may be due to the small sample size. They were, however, diagnosed with their CRC significantly quicker post completion of the FOBt. This raises the question regarding retesting at an earlier interval, or for testing with an alternate kit, such as an immunohistochemical FOBt (iFOBt), which allows for a differential cut-off level for a positive result.

It is encouraging that after a screening colonoscopy there were no missed CRCs detected. All cancers detected through a screening colonoscopy were either at the initial invite, or diagnosed at the planned surveillance colonoscopy. The rates of local excisions for Dukes' A cancers are significantly higher for those who had a screening colonoscopy. Assuming there was no systematic difference in size or morphology of Dukes' A tumours between groups, this may be a reflection on the high level of ability in performing polypectomies for those accredited colonoscopists.

In conclusion, this paper demonstrates the impact of the BCSP within one region of England since it has been rolled out nationally. The benefits of earlier stage detection and improved survival of screen-detected cancers are clear. However, there are still large numbers of cancers that are not detected through the guaiac-based FOBt. Possible reasons for this have been described above. In particular, failure of the current methods to detect rightsided cancers in women requires further research as this group comprise a significant number of patients falsely reassured by their results.

\section{ACKNOWLEDGEMENTS}

We thank Dorothy Simms, NORCCAG Audit Facilitator, and to Colin Taylor, BCSP North East Hub Analyst, for their help in data extraction.

\section{Conflict of interest}

Dr Colin Rees is a co-author on a manuscript covering a similar topic to this paper, from a different unit, as yet unpublished. All the remaining authors declare no conflict of interest.

\section{Author contributions}

Mr Michael Gill collected and analysed all the data and wrote the initial draft of the manuscript. All other authors have contributed in the design of the study and editing of the manuscript. 


\section{REFERENCES}

Arain MA, Sawhney M, Sheikh S, Anway R, Thyagarajan B, Bond JH, Shaukat A (2010) CIMP Status of interval colon cancers: another piece to the puzzle. Am J of Gastroenterol 105(5): 1189-1195

Cancer Research UK (2010) Bowel (colorectal) cancer - UK incidence statistics. Available from: http://info.cancerresearchuk.org/cancerstats/ types/bowel/incidence/\#source20

Chlebowski RT, Wactawski-Wende J, Ritenbaugh C, Hubbell FA, Ascensao J, Rodabough RJ, Rosenberg CA, Taylor VM, Harris R, Chen C, AdamsCampbell LL, White E (2004) Estrogen plus progestin and colorectal cancer in postmenopausal women. $N$ Engl J Med 350(10): 991-1004

Dray X, Camus M, Coelho J, Ozenne V, Pocard M, Marteau P (2011) Treatment of gastrointestinal angiodysplasia and unmet needs. Dig Liver Dis 43(7): 515-522

Faivre J, Dancourt V, Lejeune C, Tazi MA, Lamour J, Gerard D, Dassonville F, Bonithon-Kopp C (2004) Reduction in colorectal cancer mortality by fecal occult blood screening in a French controlled study. Gastroenterology 126(7): 1674-1680

Hardcastle J, Chamberlain J, Robinson M, Moss S, Amar S, Balfour T, James P, Mangham C (1996) Randomised controlled trial of faecal-occult-blood screening for colorectal cancer. Lancet 348(9040): 1472-1477

Kewenter J, Brevinge H, Engaras B, Haglind E, Ahren C (1994) Results of screening, rescreening, and follow-up in a prospective randomized study for detection of colorectal cancer by fecal occult blood testing. Results for 68,308 subjects. Scand J Gastroenterol 29(5): 468-473

Kronborg O, Fenger C, Olsen J, Jorgensen O, Sondergaard O (1996) Randomised study of screening for colorectal cancer with faecal-occultblood test. Lancet 348(9040): 1467-1471

Kronborg O, Fenger C, Sondergaard O, Pedersen KM, Olsen J (1987) Initial mass screening for colorectal cancer with fecal occult blood test.
A prospective randomized study at Funen in Denmark. Scand $J$ Gastroenterol 22(6): 677-686

Lee T, Rutter M, Blanks R, Moss S, Goddard A, Chilton A, Nickerson C, McNally R, Patnick J, Rees C (2012a) Colonoscopy quality measures: experience from the NHS Bowel Cancer Screening Programme. Gut 61(7): 1050-1057

Lee TJ, Clifford GM, Hull MA, Ritchie M, James P, McNally RJ, Rutter MD, Rees CJ (2012b) Aspirin users attending for NHS bowel cancer screening have less colorectal neoplasia: chemoprevention or false-positive faecal occult blood testing? Digestion 85(4): 278-281

Mandel JS, Church TR, Ederer F, Bond JH (1999) Colorectal cancer mortality: effectiveness of biennial screening for fecal occult blood. J Natl Cancer Inst 91(5): 434-437

National Bowel Cancer Audit Project (2011) National Bowel Cancer Audit Annual Report 2010. Document Ref: IC18050111. Available from: http://www.ic.nhs.uk/webfiles/Services/NCASP/audits\%20and\%20reports/ NHS_Bowel_Cancer_2010_INTERACTIVE.pdf

Pokoradi AJ, Iversen L, Hannaford PC (2011) Factors associated with age of onset and type of menopause in a cohort of UK women. Am J Obstet Gynecol 205(1): 34.e1-34.e13

Rees C, Rutter M (2010) NREG: old banger or new vehicle for research? Frontline Gastroenterol 1(1): 59-62

Sawhney M, Farrar W, Gudiseva S, Nelson D, Lederle F, Rector T, Bond J (2006) Microsatellite instability in interval colon cancers. Gastroenterology 131(6): 1700-1705

Steele RJC, McClements P, Watling C, Libby G, Weller D, Brewster DH, Black R, Carey FA, Fraser CG (2012) Interval cancers in a FOBT-based colorectal cancer population screening programme: implications for stage, gender and tumour site. Gut 61(4): 576-581

This work is published under the standard license to publish agreement. After 12 months the work will become freely available and the license terms will switch to a Creative Commons Attribution-NonCommercial-Share Alike 3.0 Unported License. 